\title{
Candida parapsilosis Prosthetic Valve Endocarditis: A Multifaceted Problem
}

\author{
Yatin Mehta $^{1} \mathbb{B}$, Vikas Deswal $^{2}$ (C) \\ Indian Journal of Critical Care Medicine (2021): 10.5005/jp-journals-10071-23941
}

In this issue of JCCC, authors from Srichitra Institute have published a retrospective analysis of 47 prosthetic valve endocarditis (PVE) of which 12 (25.5\%) were due to Candida parapsilosis.

Candida infective endocarditis (IE) is a rare disease as it accounts for $1.8 \%$ of all IE and $3.4 \%$ of all PVE. ${ }^{1,2}$ It is a very debilitating disease associated with significant morbidity and mortality. In the published case series, the mortality rate of Candida PVE is as high as $62.5 \%$. This series had a mortality of $75 \%$.

Candida PVE usually is a late complication (median time 8.9 months) of valvular surgery and nearly $50 \%$ of patients would have a prior history of IE. In this series also $58 \%$ of patients were lateonset PVE, i.e., at 26 months. A large proportion (46\%) of patients would present with systemic embolization with the majority of them having cerebral embolization, $67 \%$ of patients in this series had embolic events. ${ }^{2}$

C. parapsilosis has several virulence factors, including the ability to form biofilm on various medical devices and prosthetic material, which confer resistance to antifungal therapy and aid in the survival of the organism. ${ }^{3}$ Greater ability to form biofilm may explain the higher incidence of $C$. parapsilosis PVE.

C. parapsilosis is usually susceptible to all antifungal classes, including, azoles, polyenes, and echinocandins, although the minimum inhibitory concentration (MIC) values for echinocandins are higher than other common Candida species, which are mediated by naturally occurring polymorphism in the FKS1 gene. Repeated exposure to echinocandin is a risk factor for C. parapsilosis developing resistance. Of late there has been an alarming increase in resistance rates to both azoles and echinocandins. In a recently published study, the prevalence of multidrug-resistant (MDR) C. parapsilosis increased from 6 to $33 \%$. $^{4}$

Rapid identification and antimicrobial susceptibility pattern of infecting organisms in blood cultures are of paramount importance for diagnosing and managing complex infections, like PVE; however, the sensitivity of blood cultures for isolating Candida species was $50 \%$ among patients found to have disseminated candidiasis at autopsy. ${ }^{5}$

The diagnosis of PVE is established based on clinical manifestations, blood cultures, and echocardiography. The accepted criteria for diagnosis of IE are the modified Duke criteria. However, the sensitivity of these criteria for diagnosis of PVE is lower than their sensitivity for native valve endocarditis (NVE) given the limitation of transthoracic echocardiography (TTE), including transesophageal echocardiography (TEE).

In studies of pathologically confirmed cases of PVE involving surgically implanted valves, assessed by Duke criteria, $76 \%$ were categorized as definite PVE and $24 \%$ as possible PVE. ${ }^{6}$ The negative
${ }^{1}$ Institute of Critical Care and Anesthesia, Medanta: The Medicity, Gurugram, Haryana, India

${ }^{2}$ Department of Internal Medicine and Infectious Diseases, Medanta: The Medicity, Gurugram, Haryana, India

Corresponding Author: Yatin Mehta, Institute of Critical Care and Anesthesia, Medanta: The Medicity, Gurugram, Haryana, India, Phone: +91 9971698149, e-mail: yatinmehta@hotmail.com

How to cite this article: Mehta Y, Deswal V. Candida parapsilosis Prosthetic Valve Endocarditis: A Multifaceted Problem. Indian J Crit Care Med 2021;25(8):839-840.

Source of support: Nil

Conflict of interest: None

predictive value of the modified Duke criteria, including a complete echocardiographic evaluation, in a patient with PVE may be as low as $60-65 \%$. Based on the above evidence and recommendation by the European society of cardiology, additional imaging techniques, like 18F-FDG PET/CT, should be used in the setting of persistent clinical suspicion but "possible" or "rejected" endocarditis based on the Duke criteria. ${ }^{8}$ In this series, TEE-detected vegetation was in only one case, which was missed by TTE.

In a study on 92 patients with suspected endocarditis who were evaluated with TEE and 18F-FDG PET/CT, patients were initially classified according to the modified Duke criteria. The addition of 18F-FDG PET/CT to the modified Duke criteria as an additional major criterion was associated with increased diagnostic sensitivity for PVE (from 52 to $91 \%$ ), with a marginal drop in specificity (from 95 to 90\%), and with an increase in the negative predictive value (60-88\%). ${ }^{7}$

Based on data from various case series, the Infectious Disease Society of America (IDSA) recommends a combined antifungal and surgical approach for the management of Candida PVE., 9,10 More and more data support a combined medical-surgical approach. ${ }^{11}$ However, a large proportion of patients are deemed unfit for surgical valve replacement due to frail state, older age, and chronic medical conditions. Recommended initial therapy includes lipid/liposomal amphotericin B, 3-5 mg/kg daily, with or without flucytosine, $25 \mathrm{mg} / \mathrm{kg}$ four times daily, or a high-dose echinocandin (caspofungin $150 \mathrm{mg}$ daily, micafungin $150 \mathrm{mg}$ daily, or anidulafungin $200 \mathrm{mg}$ daily) for a minimum of 6 weeks. ${ }^{9}$

A greater amount of evidence is available for amphotericin $B$; however, there are case reports and small case series of successful treatment of Candida NVE and PVE with an echinocandin either alone or in combination with amphotericin B or azoles. ${ }^{12,13}$ Echinocandins stand out from other antifungals in their activity 
against biofilm-embedded Candida species along with fungicidal activity against the majority of clinically relevant Candida species. However, contrary to the basic science belief, the combined French-Spanish observational study found better 6 -month survival with liposomal amphotericin B-based monotherapy than in those receiving echinocandin-based monotherapy in a cohort of patients with Candida PVE. ${ }^{2}$ In this Chitra series, $83 \%$ of patients were managed with liposomal amphotericin B-based combination therapy, while $17 \%$ were managed with its monotherapy and only one patient had a surgical replacement. Echinocandins have never been systematically studied in a randomized clinical trial for Candida NVE or PVE, although the ICE study did reveal that the mortality rates were similar between the groups receiving amphotericin and an echinocandin, but this study included both NVE and PVE. ${ }^{14}$ In light of the above evidence, it may be prudent enough to use liposomal amphotericin B-based regimes for Candida PVE till more robust data are available on echinocandins. Also, $17 \%$ of patients in the present series had renal failure secondary to amphotericin $B$.

Lifelong chronic suppressive therapy with oral fluconazole (6-12 mg/kg) daily is recommended for C. parapsilosis PVE who cannot undergo valve replacement if the Candida isolate is susceptible. ${ }^{9}$ The role of long-term chronic suppressive therapy is not clear in the setting of a valve replacement; however, many clinicians still prefer a long-term suppressive antifungal therapy even when valve replacement is performed. Chronic suppressive therapy with azoles has been shown to reduce the risk of relapse. ${ }^{2}$ The relapse rate was much higher in patients with Candida PVE $(29 \%)^{2}$ than in those with Candida NVE (5.3\%). ${ }^{15,16}$

C. parapsilosis PVE presents a multifaceted problem in a lower-middle-income country (LMIC) like ours due to difficulty in establishing the diagnosis, shortage of antifungals, ever-increasing antifungal resistance, financial burden on patients, and lack of expertise in managing such a complex problem except in few specialized centers. Published literature on the subject reveals high mortality rates (57-62\%), and given the reasons mentioned above, expected mortality rates would be much higher in the LMIC than western world. A high index of suspicion, rapid diagnosis, and combined medical and surgical approach, with the involvement of a multidisciplinary team, would go a long way in reducing mortality. The incidence of $C$. parapsilosis PVE may be higher in public hospitals due to poor infection control practices and resource crunch. Other interesting findings in this study are significantly high mean vegetation size $(13.5 \mathrm{~mm})$, embolic events, and lower PCT. This is an important study throwing light on PVE due to C. parapsilosis in India.

\section{ORCID}

Yatin Mehta @ https://orcid.org/0000-0002-0888-4774

Vikas Deswal @ https://orcid.org/0000-0002-5758-2262

\section{References}

1. Falcone M, Barzaghi N, Carosi G, Grossi P, Minoli L, Ravasio V, et al. Candida infective endocarditis: report of 15 cases from a prospective multicenter study. Medicine (Baltimore) 2009;88(3):160-168. DOI: 10.1097/MD.0b013e3181a693f8.

2. Rivoisy C, Vena A, Schaeffer L, Charlier C, Fontanet A, Delahaye F, et al. Prosthetic valve Candida spp. endocarditis: new insights into long-term prognosis-the ESCAPE study. Clin Infect Dis 2018;66(6): 825-832. DOI: $10.1093 / \mathrm{cid} / \mathrm{cix} 913$.

3. Neji S, Hadrich I, Trabelsi H, Abbes S, Cheikhrouhou F, Sellami H, et al. Virulence factors, antifungal susceptibility and molecular mechanisms of azole resistance among Candida parapsilosis complex isolates recovered from clinical specimens. J Biomed Sci 2017;24(1):67. DOI: 10.1186/s12929-017-0376-2.

4. Mete B, Zerdali EY, Aygun G, Saltoglu N, Balkan II, Karaali R, et al. Change in species distribution and antifungal susceptibility of candidemias in an intensive care unit of a university hospital (10-year experience). Eur J Clin Microbiol Infect Dis 2021;40(2):325-333. DOI: 10.1007/s10096-020-03994-6.

5. Clancy CJ, Nguyen MH. Finding the "missing 50\%" of invasive candidiasis: how nonculture diagnostics will improve understanding of disease spectrum and transform patient care. Clin Infect Dis 2013;56(9):1284-1292. DOI: 10.1093/cid/cit006.

6. John MD, Hibberd PL, Karchmer AW, Sleeper LA, Calderwood SB. Staphylococcus aureus prosthetic valve endocarditis: optimal management and risk factors for death. Clin Infect Dis Off Publ Infect Dis Soc Am 1998;26(6):1302-1309. DOI: 10.1086/516378.

7. Pizzi MN, Roque A, Fernández-Hidalgo N, Cuéllar-Calabria $H$, Ferreira-González I, Gonzàlez-Alujas MT, et al. Improving the diagnosis of infective endocarditis in prosthetic valves and intracardiac devices with 18F-fluordeoxyglucose positron emission tomography/ computed tomography angiography. Circulation 2015;132(12): 1113-1126. DOI: 10.1161/CIRCULATIONAHA.115.015316.

8. Habib G, Lancellotti P, Antunes MJ, Bongiorni MG, Casalta JP, Del Zotti F, et al. 2015 ESC Guidelines for the management of infective endocarditis. Eur Heart J 2015;36(44):3075-3128. DOI: 10.1093/ eurheartj/ehv319. Available from: https://academic.oup.com/ eurheartj/article/36/44/3075/2293384.

9. Pappas PG, Kauffman CA, Andes DR, Clancy CJ, Marr KA, OstroskyZeichner $\mathrm{L}$, et al. Clinical practice guideline for the management of candidiasis: 2016 update by the Infectious Diseases Society of America. Clin Infect Dis 2016;62(4):e1-e50. DOI: 10.1093/cid/civ933.

10. Baddour LM, Wilson WR, Bayer AS, Fowler VG, Tleyjeh IM, Rybak MJ, et al. Infective endocarditis in adults: diagnosis, antimicrobial therapy, and management of complications. Circulation 2015;132(15):1435-1486. DOI: 10.1161/CIR.0000000000000296.

11. Steinbach WJ, Perfect JR, Cabell CH, Fowler VG, Corey GR, Li JS, et al. A meta-analysis of medical versus surgical therapy for Candida endocarditis. J Infect 2005;51(3):230-247.DOI: 10.1016/j.jinf.2004.10.016.

12. López-Ciudad V, Castro-Orjales MJ, León C, Sanz-Rodríguez C, de la Torre-Fernández MJ, de Juan-Romero MAP, et al. Successful treatment of Candida parapsilosis mural endocarditis with combined caspofungin and voriconazole. BMC Infect Dis 2006;6:73. DOI: 10.1186/1471-2334-6-73.

13. Lye DCB, Hughes A, O'Brien D, Athan E. Candida glabrata prosthetic valve endocarditis treated successfully with fluconazole plus caspofungin without surgery: a case report and literature review. Eur J Clin Microbiol Infect Dis 2005;24(11):753-755. DOI: 10.1007/s10096-005-0038-2.

14. Arnold CJ, Johnson M, Bayer AS, Bradley S, Giannitsioti E, Miró JM, et al. Candida infective endocarditis: an observational cohort study with a focus on therapy. Antimicrob Agents Chemother 2015;59(4):2365-2373. DOI: 10.1128/AAC.04867-14.

15. Lefort A, Chartier L, Sendid B, Wolff M, Mainardi J-L, Podglajen I, et al. Diagnosis, management and outcome of Candida endocarditis. Clin Microbiol Infect Off Publ Eur Soc Clin Microbiol Infect Dis 2012;18(4):E99-E109. DOI: 10.1111/j.1469-0691.2012.03764.x.

16. Ponnambath DK, Gopalakrishnan A, Pillai VV, Kaviyil JE, Raja K. Clinical Profile of Prosthetic Valve Endocarditis due to Candida parapsilosis: A 11-year Retrospective Observational Study from a Quaternary Cardiac Referral Institute in India. Indian J Crit Care Med 2021;25(8):857-862. 